\title{
ON A THEOREM OF FEIT AND TITS
}

\author{
PETER B. KLEIDMAN AND MARTIN W. LIEBECK
}

(Communicated by Warren J. Wong)

\begin{abstract}
Feit and Tits [3] lay the groundwork for determining the smallest degree of a projective representation of a finite extension of a finite simple group $G$. Provided $G$ is not of Lie type in characteristic 2 , they determine precisely when this degree is smaller than the degree of a projective representation of $G$ itself. We complete this project by extending their results to the groups of Lie type in characteristic 2 .
\end{abstract}

\section{INTRODUCTION}

In [3], Feit and Tits address the problem of finding the smallest degree of a nontrivial projective representation of a finite extension of a finite simple group $G$. Here, by a projective representation we mean a homomorphism to $\mathrm{PGL}_{n}(\mathbf{F})$ for some field $\mathbf{F}$ and some integer $n$, and by a finite extension of $G$ we mean a finite group with a homomorphic image isomorphic to $G$. Let $G$ be a nonabelian finite simple group and $\mathbf{F}$ an algebraically closed field, and define

$$
\begin{aligned}
& R_{\mathbf{F}}(G)=\min \left\{n \mid G \text { is contained in } \operatorname{PGL}_{n}(\mathbf{F})\right\} \\
& M_{\mathbf{F}}(G)=\min \left\{n \mid \text { a finite extension of } G \text { is contained in } \operatorname{PGL}_{n}(\mathbf{F})\right\} .
\end{aligned}
$$

The interesting situation is the case in which $M_{\mathrm{F}}(G)<R_{\mathrm{F}}(G)$, and this is the subject under study in [3]. Indeed, Feit and Tits lay the groundwork for the classification of this situtation, and the purpose of our paper is to complete this classification. This appears in Theorem 3 below.

The symplectic groups $\mathrm{Sp}_{2 n}(2)$ (with $n \geq 4$ ) provide examples in which $M_{\mathrm{F}}(G)<R_{\mathrm{F}}(G)$. Consider the group $H_{o}$ described in [5, Theorem 5(b)], so that

$$
H_{o} \cong\left(4 \circ 2^{1+2 n}\right) \cdot \mathrm{Sp}_{2 n}(2) \text {. }
$$

The proof of [5, Theorem 5(b)] shows that $H_{o}$ embeds in $\mathrm{GL}_{2^{n}}(\mathrm{~F})$ for any algebraically closed field of odd characteristic or of characteristic 0 , and hence $2^{2 n} \cdot \mathrm{Sp}_{2 n}(2)$ embeds in $\mathrm{PGL}_{2^{n}}(\mathbf{F})$. Consequently $M_{\mathbf{F}}\left(\mathrm{Sp}_{2 n}(2)\right) \leq 2^{n}$. On the other hand, it follows from [10] that $R_{\mathrm{F}}\left(\mathrm{Sp}_{2 n}(2)\right)>2^{n}$, provided $n \geq 4$.

Received by the editors September 6, 1988 and, in revised form, October 3, 1988.

1980 Mathematics Subject Classification (1985 Revision). Primary 20C25. 
Observe that if $G$ is any simple subgroup of $\operatorname{Sp}_{2 n}(2)$ and $\operatorname{char}(\mathbf{F}) \neq 2$, then $M_{\mathbf{F}}(G) \leq M_{\mathbf{F}}\left(\mathrm{Sp}_{2 n}(2)\right) \leq 2^{n}$. With this is mind, let us define

$$
n_{G}=\min \left\{n \mid G \text { embeds in } \operatorname{Sp}_{2 n}(2)\right\} \text {. }
$$

We now see that $M_{\mathbf{F}}(G) \leq \min \left\{2^{n_{G}}, R_{\mathbf{F}}(G)\right\}$, provided $\operatorname{char}(\mathbf{F}) \neq 2$. One of the fundamental results in [3] is that equality holds here.

Theorem 1 [3]. Assume that $G$ is a nonabelian simple group, $\mathbf{F}$ is an algebraically closed field, and that $M_{\mathbf{F}}(G), R_{\mathbf{F}}(G)$ and $n_{G}$ are as defined above.

(i) If $\operatorname{char}(\mathbf{F})=2$, then $M_{\mathbf{F}}(G)=R_{\mathbf{F}}(G)$.

(ii) If $\operatorname{char}(\mathbf{F}) \neq 2$, then $M_{\mathbf{F}}(G)=\min \left\{2^{n_{G}}, R_{\mathbf{F}}(G)\right\}$.

Using Theorem 1, Feit and Tits $[3, \S 4]$ then go on to show that any (then known) simple group $G$ satisfying $M_{\mathrm{F}}(G)<R_{\mathrm{F}}(G)$ must be of Lie type in characteristic 2. Quoting the classification of finite simple groups, we can now state

Theorem 2 [3, §4]. If $M_{\mathbf{F}}(G)<R_{\mathbf{F}}(G)$, then $G$ is of Lie type in characteristic 2 .

Here we analyse the simple groups of Lie type in characteristic 2, and we determine precisely which simple groups $G$ can in fact satisfy $M_{\mathrm{F}}(G)<R_{\mathrm{F}}(G)$. Our main result is

Theorem 3. Let $G$ be a nonabelian simple group and $\mathbf{F}$ an algebraically closed field. Then $M_{\mathbf{F}}(G)<R_{\mathbf{F}}(G)$ if and only if $G$ and $\mathbf{F}$ appear in the following table:

\begin{tabular}{|c|c|c|c|c|}
\hline$G$ & $\operatorname{char}(\mathbf{F})$ & $R_{\mathbf{F}}(G) \geq$ & $M_{\mathbf{F}}(G)$ & conditions \\
\hline $\mathrm{Sp}_{2 n}(q), q$ even & $\neq 2$ & $\frac{1}{2} q^{n-1}\left(q^{n-1}-1\right)(q-1)$ & $q^{n}$ & $n \geq 2$, \\
& & & & $G \neq \operatorname{Sp}_{4}(2)^{\prime}, \operatorname{Sp}_{6}(2)$ \\
$\Omega_{2 n}^{ \pm}(q), q$ even & $\neq 2$ & $q^{n-1}\left(q^{n-2}-1\right)$ & $q^{n}$ & $n \geq 4$, \\
& & & & $G \neq \Omega_{8}^{+}(2)$ \\
$L_{4}(q), q$ even & $\neq 2$ & $q^{3}+q^{2}+q-1$ & $q^{3}$ & $G \neq L_{4}(2)$ \\
$G_{2}(q), q=2^{2 e}$ & $\neq 2,3$ & $q^{3}+1$ & $q^{3}$ & $G \neq G_{2}(4)$ \\
\hline
\end{tabular}

Our strategy for showing $2^{n_{G}} \geq R_{\mathrm{F}}(G)$ (for those $G$ not in the table) is to establish lower bounds for $n_{G}$ using some modular representation theory to be found in $[10,11,12]$, and to establish upper bounds for $R_{\mathrm{F}}(G)$ by finding suitably small representations appearing in $[1,2,7,13]$. To prove that $2^{n_{G}}<$ $R_{\mathrm{F}}(G)$ (for those $G$ in the table) is for the most part straightforward, although we have to appeal to some results of $[4,6,9,14]$ in order to obtain lower bounds for $R_{\mathrm{F}}(G)$. In $\S 2$ we take care of various technical details which provide the bounds we require. The proof of Theorem 3 is then completed in $\S 3$. 


\section{BoundS FOR $n_{G}$ AND $R_{\mathrm{F}}(G)$}

Throughout this section, $G=G\left(2^{a}\right)=G(q)$ denotes a simple group of Lie type over the field $\mathbf{F}_{2^{a}}=\mathbf{F}_{q}$, and $\mathbf{F}$ denotes an algebraically closed field. As a convenience, we sometimes write $L_{d}^{+}(q)=L_{d}(q), L_{d}^{-}(q)=U_{d}(q), E_{6}^{+}(q)=$ $E_{6}(q)$ and $E_{6}^{-}(q)={ }^{2} E_{6}(q)$.

Proposition 4. Write $d_{G}$ for the smallest nontrivial 2-modular projective representation of $G$. Then $d_{G}$ appears in the following table:

\begin{tabular}{cc}
$G$ & $d_{G}$ \\
\hline$L_{d}^{ \pm}(q)$ & $d$ \\
$\mathrm{Sp}_{d}(q)^{\prime}, d \geq 4$ & $d$ if $G \neq \mathrm{Sp}_{4}(2)^{\prime}$ \\
$\Omega_{d}^{ \pm}(q), d \geq 8$ & 3 if $G=\mathrm{Sp}_{4}(2)^{\prime}$ \\
$G_{2}(q)^{\prime}$ & $d$ \\
${ }^{2} B_{2}(q)$ & 6 \\
${ }^{3} D_{4}(q)$ & 4 \\
$F_{4}(q),{ }^{2} F_{4}(q)^{\prime}$ & 8 \\
$E_{6}^{ \pm}(q)$ & 26 \\
$E_{7}(q)$ & 27 \\
$E_{8}(q)$ & 56
\end{tabular}

Proof. This is well known. A proof can be found in [12, §2].

Assume that $V$ is an $\mathbf{E} G$-module for some field $\mathbf{E}$. For a subfield $\mathbf{E}_{o}$ of $\mathbf{E}$, we say that $V$ is realized over $\mathbf{E}_{o}$ if $G$ acts on the $\mathbf{E}_{o}$-span of some $\mathbf{E}$-basis of $V$. In other words, the matrices corresponding to elements of $G$ can be written with entries in $\mathbf{E}_{o}$.

Proposition 5. Let $V$ be an absolutely irreducible $\mathbf{F}_{2^{b}} G$-module which is realized over no proper subfield of $\mathbf{F}_{2^{b}}$, and let $d=d_{G}$ be as in Proposition 4.

(i) If $G$ is untwisted or if $G$ is of type ${ }^{2} B_{2}$ or ${ }^{2} F_{4}$, then $b \mid a$ and $\operatorname{dim}_{\mathbf{F}_{2 b}}(V) \geq d^{a / b}$.

(ii) If $G \cong U_{d}\left(2^{a}\right), \Omega_{d}^{-}\left(2^{a}\right)$ or ${ }^{2} E_{6}\left(2^{a}\right)$, then $b \mid 2 a$ and one of the following holds.

(a) $b+a$ and $\operatorname{dim}_{\mathrm{F}_{2 b}}(V) \geq d^{2 a / b}$.

(b) $b \mid a$ and $\operatorname{dim}_{\mathrm{F}^{b}}(V) \geq d^{a / b}$.

Proof. Let $k$ denote the algebraic closure of $\mathbf{F}_{2}$, and form the tensor product $\bar{V}=V \otimes k$. There is a corresponding homomorphism $\rho$ from $G$ to $\mathrm{GL}_{m}(k)$, where $m=\operatorname{dim}_{k}(\bar{V})=\operatorname{dim}_{\mathrm{F}_{2 b}}(V)$. Write $\nu$ for the natural automorphism 
of $\mathrm{GL}_{m}(k)$ given by $t \mapsto t^{2}$ on matrix entries. Composing $\rho$ with $\nu^{i}(i=$ $0,1,2, \ldots)$ gives rise to modules which we call $V^{(i)}$. Since the $k G$-module $\bar{V}$ is realized over $\mathbf{F}_{2^{b}}$, we have $\bar{V} \cong \bar{V}^{(b)}$. And since $V$ is realized over no proper subfield of $\mathbf{F}_{2^{b}}$, whenever $\bar{V} \cong \bar{V}^{(i)}$, it follows that $b \mid i$.

Suppose first that $G$ is untwisted or is of type ${ }^{2} B_{2}$ or ${ }^{2} F_{4}$. Then according to [15, p. 241], $\mathbf{F}_{2^{a}}$ is a splitting field for $G$. This means that $\bar{V}$ is realized over $\mathbf{F}_{2^{a}}$, and by the remark in the previous paragraph, $b \mid a$. It now follows from Theorems 2.1 and 2.3 in [11] that $m \geq d^{a / b}$, and so (i) is proved.

Now take the case where $G \cong U_{d}\left(2^{a}\right), \Omega_{d}^{-}\left(2^{a}\right)$ or ${ }^{2} E_{6}\left(2^{a}\right)$. Here [15, p. 241] implies that $\mathbf{F}_{2^{2 a}}$ is a splitting field, whence $b \mid 2 a$. Obviously $a /(a, b)=a / b$ if $b \mid a$, and $a /(a, b)=2 a / b$ if $b \nmid a$, and so (ii) follows directly from Theorem 2.2 of [11].

We are now in a position to obtain lower bounds for $n_{G}$. In the proof of Proposition 6 below, it is convenient to quote the theorem of Zsigmondy [16] which says the following. Let $x, y$ be integers with $x \geq 2$ and $y \geq 3$. Then provided $(x, y) \neq(2,6)$, there exists a prime divisor $p$ of $x^{y}-1$ such that $p$ does not divide $x^{z}-1$ for $1 \leq z \leq y-1$. Such a prime is called a primitive prime divisor of $x^{y}-1$.

Proposition 6. Let $d=d_{G}$ be as in Proposition 4. A lower bound for $n_{G}$ appears in the following table:

\begin{tabular}{cc}
$G$ & $n_{G} \geq$ \\
\hline$L_{2}\left(2^{a}\right)$ & $a$ \\
$L_{d}^{ \pm}\left(2^{a}\right), d \geq 3$ & da if $d \neq 4$ \\
$\mathrm{Sp}_{d}\left(2^{a}\right)^{\prime}, d \geq 4$ & $3 a$ if $d=4$ \\
$\Omega_{d}^{ \pm}\left(2^{a}\right), d \geq 8$ & $d a / 2$ \\
$G_{2}\left(2^{a}\right)^{\prime}$ & $3 a / 2$ \\
${ }^{2} B_{2}\left(2^{a}\right)$ & $2 a$ \\
${ }^{3} D_{4}\left(2^{a}\right)$ & $6 a$ \\
$F_{4}\left(2^{a}\right),{ }^{2} F_{4}\left(2^{a}\right)^{\prime}$ & $13 a$ \\
$E_{6}^{ \pm}\left(2^{a}\right)$ & $27 a / 2$ \\
$E_{7}\left(2^{a}\right)$ & $28 a$ \\
$E_{8}\left(2^{a}\right)$ & $124 a$
\end{tabular}

Proof. Write $G \leq \operatorname{Sp}_{2 n_{G}}(2)$, so that $G$ acts on the natural $2 n_{G}$-dimensional module over $\mathrm{F}_{2}$. Let $V$ be a $G$-invariant section of this module upon which $G$ 
acts faithfully and irreducibly. Write $m=\operatorname{dim}_{\mathbf{F}_{2}}(V)$, so that $G \leq \mathrm{GL}\left(V, \mathbf{F}_{2}\right) \cong$ $\mathrm{GL}_{m}(2)$. Now there is a divisor $b$ of $m$ such that as an $\mathbf{F}_{2^{b}} G$-module,

$$
V \otimes \mathbf{F}_{2^{b}} \cong \bigoplus_{\sigma \in \operatorname{Gal}\left(\mathbf{F}_{2^{b}}: \mathbf{F}_{2}\right)} W^{\sigma},
$$

where $W$ is an absolutely irreducible $\mathbf{F}_{2^{b}} G$-module with $\operatorname{dim}_{\mathbf{F}_{2^{b}}}(W)=m / b$ (see [8, Theorem 9.21] for example). Moreover, if $\sigma, \tau \in \operatorname{Gal}\left(\mathbf{F}_{2^{b}}: \mathbf{F}_{2}\right)$ with $\sigma \neq \tau$, then $W^{\sigma} \not W^{\tau}$ as $\mathbf{F}_{2^{b}}$-modules, and it follows that $W$ is realized over no proper subfield of $\mathbf{F}_{2^{b}}$. We are now in a position to exploit Proposition 5 .

First assume that $G$ is of type $L_{2}, \mathrm{Sp}_{d}, \Omega_{d}^{+}, G_{2},{ }^{2} B_{2},{ }^{2} F_{4}, F_{4}, E_{6}, E_{7}$ or $E_{8}$. Then it follows from Proposition 5 that $b \mid a$ and $m / b \geq d^{a / b}$. Hence $2 n_{G} \geq$ $m \geq b d^{a / b} \geq d a$, as required.

Assume here that $G \cong L_{d}\left(2^{a}\right)$ with $d \geq 3$. As in the previous paragraph, we know that $b \mid a$. Consider first the case in which $a=b$. Here $m / a=m / b \geq$ $d_{G}=d$. Now if $V$ is not a self-dual $G$-module, it follows that $2 n_{G} \geq 2 m \geq$ $2 d a$, as desired. We may assume therefore that $V$ is self-dual, and hence so is $V \otimes \mathbf{F}_{2^{a}}$. Consequently $W^{*} \cong W^{\sigma}$ for some $\sigma \in \operatorname{Gal}\left(\mathbf{F}_{2^{a}}: \mathbf{F}_{2}\right)$, and hence $W$ is either self-dual or unitary according as $|\sigma|=1$ or 2 . Since the irreducible $d$-dimensional modules for $\mathrm{SL}_{d}\left(2^{a}\right)$ are neither self-dual or unitary, we have $m / a>d$. Therefore by [11, Theorem 1.1], $m / a \geq \frac{1}{2} d(d-1)$. So if $d \geq 5$, then $m \geq 2 d a$, and the desired result follows. If $d=4$, we have $m \geq 6 a$, whence $n_{G} \geq 3 a$. If $d=3$, then it follows from [12, Theorem 2.2] that there are no irreducible $\mathbf{F}_{2^{b}} L_{3}\left(2^{a}\right)$-modules of dimension 4 or 5 , and so once again $m \geq 6 a$, which proves $n_{G} \geq 3 a$. We now consider the case where $b<a$. Here $m / b \geq d^{a / b}$, and so with only one exception, $m \geq b d^{a / b} \geq 2 d a$, as desired. The one exception is the case $d=3$ and $a=2 b$. To treat this exceptional case, observe that the proof of Proposition 5(i) (see the proof of [11, Theorem 2.1]) actually shows that $W \otimes \mathbf{F}_{2^{a}} \cong U \otimes U^{\tau}$, where $U$ is an absolutely irreducible $\mathbf{F}_{2^{a}} \mathbf{S L}_{3}\left(2^{a}\right)$-module and $\tau$ generates $\operatorname{Gal}\left(\mathbf{F}_{2^{a}}: \mathbf{F}_{2^{b}}\right)$. Write $d_{1}=\operatorname{dim}_{\mathbf{F}_{2^{a}}}(U)$, so that $m / b=d_{1}^{2}$. If $d_{1} \geq 4$, then $2 n_{G} \geq m \geq 16 b=8 a>6 a=2 d a$, which is the required result. And if $d_{1}=3$, then $U$ is neither self-dual nor unitary, and hence the same holds of $W$; hence as we argued before, $V$ is not self-dual. Therefore $n_{G} \geq m \geq 9 b=9 a / 2>3 a$, as claimed.

Next suppose that $G \cong \Omega_{d}^{-}\left(2^{a}\right)$, with $d$ even and $d \geq 8$, so that $b \mid 2 a$ by Proposition 5. If $b$ does not divide $a$, then $m / b \geq d^{2 a / b}$, and so $m \geq 2 a d$, as desired. And if $b \mid a$, then as before we obtain $m / b \geq d^{a / b}$, and hence $m \geq d a$.

Assume here that $G \cong U_{d}\left(2^{a}\right)$ with $d \geq 3$. If $d=3$, then $a \geq 2$ (since $U_{3}(2)$ is solvable), and $2^{3 a}+1|| G \mid$. Thus $|G|$ is divisible by a primitive prime divisor of $2^{6 a}-1$, and so $m \geq 6 a$, as claimed. We assume for the rest of this paragraph that $d \geq 4$. By Proposition 5, $b \mid 2 a$. Moreover, if $b$ does not divide $a$, we may argue as in the previous paragraph. Assume therefore that $b \mid a$. If $b<a$, then $m / b \geq d^{a / b}$, and so $m \geq b d^{a / b} \geq 2 d a$ (since $d \geq 4$ ). It remains 
to consider the case $a=b$. Then by [11, Theorem 2.2], we have

$$
m / b=m / a \geq \begin{cases}d(d-1) & \text { if } d \geq 7 \\ 20 & \text { if } 5 \leq d \leq 6 \\ 6 & \text { if } d=4 .\end{cases}
$$

So provided $d \geq 5$, we have $m / a>2 d$, whence $n_{G}>d a$. When $d=4$, we deduce $m / a \geq 6$, whence $n_{G} \geq 3 a$.

If $G \cong{ }^{2} E_{6}\left(2^{a}\right)$, then as in the previous two paragraphs, we see that $m \geq$ $27 a$, and so $n_{G} \geq 27 a / 2$.

Finally, assume that $G \cong{ }^{3} D_{4}\left(2^{a}\right)$. Then $2^{4 a}-2^{2 a}+1|| G \mid$, and so $G$ is divisible by a primitive prime divisor of $2^{12 a}-1$. This forces $m \geq 12 a$, and the result follows.

At this stage we provide upper bounds for $R_{\mathrm{F}}(G)$.

Proposition 7. Apart from $\mathrm{Sp}_{d}(q)$ and $\Omega_{d}^{ \pm}(q)$, upper bounds for $R_{\mathbf{F}}(G)$ are given in the following table:

\begin{tabular}{cc}
$G$ & $R_{\mathrm{F}}(G) \leq$ \\
\hline$L_{d}^{ \pm}(q)$ & $\left(q^{d}-1\right) /(q \mp 1)$ \\
$G_{2}(q)^{\prime}$ & $q^{3}+\varepsilon, \varepsilon= \pm 1, q \equiv \varepsilon(\bmod 3)$ \\
${ }^{2} B_{2}(q)$ & $\sqrt{q / 2}(q-1)$ \\
${ }^{3} D_{4}(q)$ & $q\left(q^{4}-q^{2}+1\right)$ \\
${ }^{2} F_{4}(q)^{\prime}$ & $\sqrt{q / 2}\left(q^{2}-1\right)\left(q^{3}+1\right)$ \\
$F_{4}(q)$ & $\frac{1}{2} q(q+1)^{2}\left(q^{2}-q+1\right)^{2}\left(q^{4}+1\right)$ \\
$E_{6}^{ \pm}(q)$ & $q\left(q^{4} \mp 1\right)\left(q^{6} \pm q^{3}+1\right)$ \\
$E_{7}(q)$ & $q\left(q^{6}+q^{5}+q^{4}+q^{3}+q^{2}+q+1\right) \times$ \\
& $\left(q^{4}-q^{2}+1\right)\left(q^{6}-q^{5}+q^{4}-q^{3}+q^{2}-q+1\right)$ \\
$E_{8}(q)$ & $q\left(q^{2}+1\right)^{2}\left(q^{4}+1\right)\left(q^{4}-q^{2}+1\right) \times$ \\
& $\left(q^{8}-q^{6}+q^{4}-q^{2}+1\right)\left(q^{8}-q^{4}+1\right)$
\end{tabular}

Proof. It suffices to exhibit a faithful ordinary character of a covering group of $G$ which has degree less than or equal to the number given in the righthand column. For $L_{d}(q)$ this is easy, as it has a permutation representation of degree $\left(q^{d}-1\right) /(q-1)$, and hence has a faithful ordinary character of degree 1 less. The result for $U_{d}\left(2^{a}\right)$ appears in [13], and for the exceptional groups apart from $G_{2}(q)$ one can appeal to [1, §13.9]. For $G_{2}(q)$ we appeal to [2] or [14, pp. 293-4].

\section{THE PROOF OF THEOREM 3}

Assume initially that $G$ and $\mathbf{F}$ are as given in the table appearing in the statement of Theorem 3. We must show that $M_{\mathrm{F}}(G)<R_{\mathrm{F}}(G)$, and to do so 
it suffices to verify the values of $M_{\mathrm{F}}(G)$ and the bounds for $R_{\mathrm{F}}(G)$ displayed in the table. The bounds for $R_{\mathrm{F}}(G)$ when $G$ is $\operatorname{Sp}_{2 n}(q)$ or $\Omega_{2 n}^{\varepsilon}(q)$ are taken from [10]. When $G=L_{4}(q)$ with $q \geq 4$, it follows from [4] that the smallest ordinary faithful character degree of $G$ is $q\left(q^{2}+q+1\right)$. It can be seen using the decomposition matrices for $\mathrm{GL}_{4}(q)$ in [9] that $R_{\mathrm{F}}(G) \geq q\left(q^{2}+q+1\right)-1$. And when $G$ is $G_{2}(q), q=4^{k}>4$, it is shown in $[6,14]$ that $R_{\mathbf{F}}(G)=q^{3}+1$ (n.b., $\operatorname{char}(\mathbf{F}) \geq 5)$. Now when $G=\operatorname{Sp}_{2 n}(q)$ with $q=2^{a}$, it is obvious that $G<$ $\operatorname{Sp}_{2 n a}(2)$, and so $n_{G} \leq n a$. Consequently $n_{G}=n a$ by Proposition 6 , and as $2^{n_{G}}=q^{n}<R_{\mathrm{F}}(G)$, we deduce from Theorem 1 that $M_{\mathbf{F}}(G)=q^{n}$. In the exact same fashion we may establish $M_{\mathrm{F}}\left(\Omega_{2 n}^{ \pm}(q)\right)=q^{n}$ for $n \geq 4$. Also $L_{4}\left(2^{a}\right) \cong$ $\Omega_{6}^{+}\left(2^{a}\right)<\operatorname{Sp}_{6}\left(2^{a}\right)<\operatorname{Sp}_{6 a}(2)$ and $G_{2}\left(2^{a}\right)<\operatorname{Sp}_{6 a}(2)$, and hence by Proposition 6 we have $n_{G}=3 a$ for these groups. Thus $M_{\mathbf{F}}\left(L_{4}(q)\right)=M_{\mathbf{F}}\left(G_{2}(q)\right)=q^{3}$.

We now prove the converse. Assume that $G$ is a nonabelian simple group, F an algebraically closed field, and assume that $M_{\mathrm{F}}(G)<R_{\mathrm{F}}(G)$. Our goal is to show that $G$ and $\mathbf{F}$ appear in the table given in Theorem 3. Now according to Theorem 1, $\operatorname{char}(\mathbf{F}) \neq 2$ and

$$
2^{n_{G}}<R_{\mathbf{F}}(G)
$$

Also, Theorem 2 asserts that $G$ is of Lie type in characteristic 2 . Suppose first that $G \cong \operatorname{Sp}_{2 n}(q)^{\prime}$ with $n \geq 2$. As $R_{\mathbf{F}}\left(\operatorname{Sp}_{4}(2)^{\prime}\right) \leq 3$ and $R_{\mathbf{F}}\left(\operatorname{Sp}_{6}(2)\right)=7$, it follows that $G$ is not one of these, and so $G$ does indeed appear in the table. And if $G \cong \Omega_{2 n}^{ \pm}(q)$ with $n \geq 4$, then $G$ is not $\Omega_{8}^{+}(2)$, for $R_{\mathbf{F}}\left(\Omega_{8}^{+}(2)\right)=8$, while $n_{\Omega_{8}^{+}(2)}=4$. So once again, $G$ appears in the table. Thus we can assume hereafter that $G$ is neither a symplectic group nor an orthogonal group. It now follows from $(*)$ and Propositions 6 and 7 that $G$ is $L_{2}(q), L_{4}(q)$ or $G_{2}(q)$. Now if $G \cong L_{2}(q)$, then $2^{n_{G}}=q$; however it is well known that $R_{\mathrm{F}}(G) \leq q-1$. Thus this case cannot arise. And if $G \cong L_{4}(2)$, then $2^{n_{G}}=8$, while $R_{\mathrm{F}}(G) \leq 7$. So this case cannot arise either, and so $G$ does indeed appear in the table if $G \cong L_{4}(q)$. It remains to consider $G \cong G_{2}(q)$, so that $2^{n_{G}}=q^{3}$ by Proposition 6. It follows from [2] that if $\log _{2}(q)$ is odd, then $R_{\mathrm{F}}(G) \leq q^{3}-1$. Thus it must be the case that $\log _{2}(q)$ is even. The group $G_{2}(4)$ has an exceptional multiplier and the covering group 2.G $G_{2}(4)$ has a faithful representation of degree 14. Thus we must have $G \cong G_{2}\left(2^{2 e}\right)$ with $e \geq 2$. Now according to [7], $R_{\mathrm{F}}(G) \leq q^{3}$ if $\operatorname{char}(\mathbf{F})=3$, and we conclude that $\operatorname{char}(\mathbf{F}) \neq 2,3$. Thus the proof is finally complete.

\section{ACKNOWLEDGMENT}

The authors thank Professor G. Hiss for communicating to us his results on the modular representations of $G_{2}(q)$. 


\section{REFERENCES}

1. R. W. Carter, Finite groups of Lie type, Conjugacy Classes and Complex Characters, J. Wiley and Sons, 1985.

2. H. Enomoto and H. Yamada, The characters of $G_{2}\left(2^{n}\right)$, Japan J. Math. (N.S.) 12 (1986), 325-377.

3. W. Feit and J. Tits, Projective representations of minimum degree of group extensions, Canad. J. Math. 30 (1978), 1092-1102.

4. J. A. Green, The characters of the finite general linear groups, Trans. Amer. Math. Soc. 80 (1955), 402-447.

5. R. L. Griess, Jr., Automorphisms of extra special groups and nonvanishing degree 2 cohomology, Pacific J. Math. 48 (1973), 403-422.

6. G. Hiss, On the decomposition numbers of $G_{2}(q)$, J. Algebra 120 (1989), 339-360.

7. G. Hiss and J. Shamash, The 3-modular representations of $G_{2}\left(2^{n}\right)$ (to appear).

8. I. M. Isaacs, Character theory of finite groups, Academic Press, New York, 1976.

9. G. D. James, The decomposition matrices for $\mathrm{GL}_{n}(q), n \leq 10$, Imperial College, London, (preprint).

10. V. Landazuri and G. M. Seitz, On the minimal degrees of projective representations of the finite Chevalley groups, J. Algebra 32 (1974), 418-443.

11. M. W. Liebeck, On the orders of maximal subgroups of the finite classical groups, Proc. London Math. Soc. (3) 50 (1985), 426-446.

12. M. W. Liebeck, The affine permutation groups of rank three, Proc. London Math. Soc. (3) 54 (1987), 447-516.

13. G. M. Seitz, Some representations of classical groups, J. London Math. Soc. 10 (1975), 115120.

14. J. Shamash, Blocks and Brauer trees for groups of type $G_{2}(q)$, Proc. Sympos. Pure Math. 47 Part 2 (1987), 283-295.

15. R. Steinberg, Lectures on Chevalley groups, Yale University Mathematics Department, 1968.

16. K. Zsigmondy, Zur theorie der Potenzreste, Monatsh. Mat. und Phys. 3 (1892), 265-284.

Department of Mathematics, California Institute of Technology, Pasadena, CALIFORNia 91125

Department of Mathematics, Imperial College of Science and Technology, London, SW7 2AZ ENGLAND 